\title{
Serum level of fibroblast growth factor 21 predicts long-term prognosis in patients with both diabetes mellitus and coronary artery calcification
}

\author{
Feng Gan ${ }^{1,2}$, Jiafang Huang ${ }^{3}$, Tianran Dai ${ }^{2}$, Maorong Li $^{2}$, Jinghua Liu ${ }^{1}$ \\ ${ }^{1}$ Department of Cardiology, Beijing Anzhen Hospital, Capital Medical University, Beijing Institute of Heart, Lung, and Blood Vessel Diseases, \\ Beijing 100029, China; ${ }^{2}$ Department of Cardiology, ${ }^{3}$ Laboratory Department, Beijing Aerospace General Hospital, Beijing 100076, China \\ Contributions: (I) Conception and design: F Gan, J Liu; (II) Administrative support: J Huang; (III) Provision of study materials: T Dai; (IV) Collection \\ and assembly of data: M Li; (V) Data analysis and interpretation: F Gan, J Huang; (VI) Manuscript writing: All authors; (VII) Final approval of \\ manuscript: All authors. \\ Correspondence to: Jinghua Liu. Department of Cardiology, Beijing Anzhen Hospital, Capital Medical University, Beijing Institute of Heart, Lung, and \\ Blood Vessel Diseases, Beijing 100029, China. Email: 1jh2000bj@126.com.
}

\begin{abstract}
Background: This study aimed to investigate the relationship between serum level of fibroblast growth factor 21 (FGF-21) and long-term prognosis in patients with both diabetes mellitus (DM) and coronary artery calcification (CAC).

Methods: The study included 1,132 patients with DM and CAC according to inclusion and exclusion criteria. Based on the baseline serum level of FGF-21, patients were divided into four groups (283 in each group): low-FGF-21 group (LFG), lower-medium-FGF-21 group (LMFG), higher-medium-FGF-21 group (HMFG), and high-FGF-21 group (HFG). Major adverse cardiovascular events (MACEs), including coronary revascularization, acute coronary syndrome (ACS), heart failure (HF), malignant arrhythmia, and sudden cardiac death (SCD), were recorded. Renal function, serum level of NT-proBNP, and left ventricular function were watched and observed during follow-up.

Results: All patients were followed up for 1.5-5.1 (2.7 \pm 2.2$)$ years. The range of baseline serum level of FGF-21 was $67.5-314.7 \mathrm{pg} / \mathrm{mL}$. The serum level of FGF-21 was $\leq 103.8 \mathrm{pg} / \mathrm{mL}$ in LFG, 108.6-184.9 pg/mL in LMFG, 199.3-271.2 pg/mL in LHFG, and $>276.1 \mathrm{pg} / \mathrm{mL}$ in HFG. The baseline CAC score (CACS) was 83.2-524.9 and the mean CACS was $124.6 \pm 37.5,186.8 \pm 51.9,271.3 \pm 62.7$, and $349.2 \pm 80.6$, respectively. During follow-up, 481 patients underwent percutaneous coronary intervention (PCI) with 71, 107, 141, and 162 in subgroups, respectively. Malignant arrhythmia occurred in 89 patients, HF in 127, and SCD in 9. At the end of the 1-year follow-up, the average eGFR, NT-proBNP, and left ventricular ejection fraction (LVEF) differed significantly among groups.
\end{abstract}

Conclusions: Lower baseline serum level of FGF-21 is a prediction for a better long-term prognosis.

Keywords: Coronary artery calcification (CAC); coronary heart disease; diabetes mellitus (DM); fibroblast growth factor 21 (FGF-21); prognosis

Submitted Nov 18, 2019. Accepted for publication Mar 09, 2020.

doi: $10.21037 /$ apm.2020.03.28

View this article at: http://dx.doi.org/10.21037/apm.2020.03.28

\section{Introduction}

Fibroblast growth factor 21 (FGF-21) is one of the FGF-19 subfamily members and can be expressed in a lot of organs and tissues, including heart and vessels in the cardiovascular system, nerves and brain, muscles, kidney, and fatty tissues.
FGF-21 among the FGF family members has become a research focus. Different from other FGF members, FGF21 has no positive effect on the growth of fibroblasts and cannot bind with unfractionated heparin specifically (1). Early studies revealed that the main function of FGF-21 
was to regulate the metabolic process systematically. Recent studies showed that FGF-21 could inhibit ventricular hypertrophy, alleviate inflammation, and improve the metabolism of cardiac tissue. Also, FGF-21 plays an important inhibitory role in the progression of coronary atherosclerosis and plaque formation of artery stenosis. Earlier studies showed that FGF-21 was critical in coronary calcification through multiple molecular mechanisms $(2,3)$. Clinical studies also had some findings. FGF-21 was associated with acute myocardial infarction (AMI), hypertension, heart failure (HF), among many others (4). With these results, the predictive value of FGF-21 for prognosis is still controversial. On the contrary, patients with diabetes mellitus (DM) often have coronary calcification, which is a risk factor for patients with coronary heart disease (CHD) (5). Based on the fact that FGF-21 was involved in both DM and coronary artery calcification (CAC) in a previous study, it was hypothesized in this study that FGF-21 had a strong correlation with the prognosis among patients with both DM and CAC at the same time.

\section{Methods}

\section{Study population and groups}

Consecutive patients who met the inclusion and exclusion criteria were enrolled between October 2013 and February 2018 in the center. These patients were diagnosed with type 2 DM (T2DM) and underwent coronary computed tomographic angiography (CCTA) to evaluate the patency of the coronary artery with suspicion of CHD. The inclusion criteria were as follows: aged more than 18 years, CAC detected by CCTA, life expectancy more than at least one year, ability to complete yearly follow-up for at least one year, no previous history of percutaneous coronary intervention (PCI), and signed the informed content profile. The exclusion criteria were as follows: malignant tumor, connective tissue diseases, active liver diseases; eGFR less than $60 \mathrm{~mL} / \mathrm{min} / 1.73 \mathrm{~m}^{2}$ or dialysis; previous history of PCI, cardiac surgery, or other micro traumatic cardiac intervention; and emergent PCI administered at the same time of enrollment. After enrollment, the patients were divided into four groups based on the level of FGF-21.

\section{Definition}

T2DM, CHD, AMI, and HF were all diagnosed according to a previously published guideline. The CAC was defined as high-density signal findings of the coronary artery in the CCTA results. Malignant arrhythmia referred to malignant ventricular premature beat, ventricular tachycardia, ventricular flutter, and ventricular fibrillation verified by 24-h Holter or random electrocardiogram [not including sudden cardiac death $(\mathrm{SCD})]$.

\section{Blood tests}

Routine blood test, liver function, renal function, electrolytes including $\mathrm{K}$ and $\mathrm{Na}$, total cholesterol, triglyceride, low-density lipoprotein cholesterol, highdensity lipoprotein cholesterol, fasting glucose, NTproBNP, and HbAlc were examined at enrollment and follow-up. Blood for the FGF-21 test was acquired at enrollment and stored at $-80^{\circ} \mathrm{C}$ after centrifugation. At the end of this study, FGF-21 was evaluated for each patient by enzyme-linked immune absorption using a kit bought from TaKaRa. The eGFR was calculated using the CKD-EPI formula.

\section{$C A C$}

CCTA was conducted at once before enrollment using the SOMATOM Definition Dual-Source computed tomography scanner. Two doctors reviewed images. The CAC score (CACS) was determined using the Agatston scoring system. CACS less than 10 was normal.

\section{Echocardiography}

Cardiac functional and structural parameters, including left ventricular ejection fraction (LVEF), left ventricular enddiastolic volume, were measured by echocardiography at enrollment and after one year.

\section{Follow-up}

All patients were followed up for at least one year. The major adverse cardiovascular events (MACEs), including coronary revascularization, acute coronary syndrome (ACS), HF, malignant arrhythmia, and SCD, were recorded.

\section{Statistical analysis}

Continuous variables were expressed as mean \pm standard deviation and compared using an unpaired, two-sided 
Student $t$-test when normal distribution and equal dispersion were confirmed. The Mann-Whitney $U$ test and the Wilcoxon's signed-rank test were used when the variance was unequal. Categorical variables were expressed as percentages (\%) and compared using the $\chi^{2}$ analysis or Fisher exact test if necessary. A P value of $<0.05$ was considered statistically significant. All statistical analyses were performed using SPSS (version 17.0 for Windows, SPSS, Inc., IL, USA).

\section{Results}

\section{Baseline characteristics}

According to the inclusion and exclusion criteria, 1,132 patients with both DM and CAC, were enrolled. The age of the patients ranged from 42 to 74 years with a mean age of $(54.2 \pm 13.7)$ years. History of DM ranged from 2.4 years to 18.3 years with a mean time of $(6.5 \pm 5.1)$ years. The baseline serum level of FGF-21 ranged from 67.5 to $344.7 \mathrm{pg} / \mathrm{mL}$ with a mean level of $(174.1 \pm 53.2) \mathrm{pg} / \mathrm{mL}$. Patients were divided into four groups: low-FGF-21 group (LFG) with FGF-21 level $\leq 103.8 \mathrm{pg} / \mathrm{mL}$, lower-mediumFGF-21 group (LMFG) with FGF-21 level from 108.6 to $184.9 \mathrm{pg} / \mathrm{mL}$, higher-medium-FGF-21 group (HMFG) with FGF-21 level from 199.3 to $271.2 \mathrm{pg} / \mathrm{mL}$, and highFGF-21 group (HFG) with FGF-21 level $\geq 276.1 \mathrm{pg} / \mathrm{mL}$. The baseline CACS ranged from 83.2 to 524.9 , with a mean score of $(233.0 \pm 91.6)$. CACS differed significantly among subgroups $(\mathrm{P}<0.05)$. Parameters, including age, gender, fasting glucose, HbA1c, total cholesterol, and others, had no statistically significant difference among the four subgroups. Parameters such as serum level of NT-proBNP and LVEF differed among subgroups, but not significantly. The patients were followed up for 1.6-5.1 years with a mean duration of $(2.7 \pm 2.2)$ years (Table 1$)$.

\section{Parameters at 1-year follow-up}

At the end of the 1-year follow-up after enrollment, some blood tests and examinations were repeated. Compared with the values at enrollment, both eGFR $(95.7 \pm 21.9 v s$. 113.4 $\pm 24.7, \mathrm{P}<0.05)$ and LVEF $(49.5 \pm 12.6$ vs. $52.2 \pm 13.8)$ values decreased significantly in general, and significant difference existed among subgroups (details listed in Table 2). HF within a 1-year follow-up was diagnosed in 127 patients $(11.2 \%)$ based on the serum levels of NT-proBNP and LVEF.

\section{MACEs in the follow-up}

In the follow-up, 481 (42.5\%) patients underwent percutaneous coronary stent implantation, while the other 651 patients received medication therapy. Malignant arrhythmia occurred in 89 (7.9\%) patients, 127 (11.2\%) patients suffered from HF, and $9(0.8 \%)$ patients died suddenly. Details are listed in Table 3. Table 3 shows that LFG had a better 1-year prognosis.

\section{Discussion}

FGF-21 has become a medical research focus in recent years. It aims at multiple targets and plays a protective role in health. Earlier studies focused on molecular mechanisms involved in physiological processes, such as calcification of artery, reticulum stress-induced apoptosis, and others (6-9). Later, some studies found some connection of circulating FGF-21 with sports, metabolism, hypertension, atherosclerosis, and even microRNA regulation in HF. Few studies evaluated the predictive value of diseases. In the present study, the baseline serum level of FGF-21 had a strong relationship with long-term prognosis. Accurately, the lower baseline serum level of FGF-21 predicted better long-term prognosis in patients with both DM and CAC. In detail, the incidence of MACEs, including coronary revascularization, ACS, HF, and malignant arrhythmia, was lower in patients with lower baseline serum levels of FGF21 compared with those with a higher level of FGF-21. These findings were not consistent with the results of the Multi-Ethnic Study of Atherosclerosis (MESA) published in Atherosclerosis by MIKE et al. MESA revealed that the serum level of FGF-21 had no overt relationship with intima-media thickness of the carotid artery, ankle-brachial index, and coronary calcification. Even after multiple factors were adjusted, no statistically significant relationship was found between the FGF-21 level and cardiovascular events. Therefore, it was concluded that FGF-21 could not be used as a risk factor for patients without a history of cardiovascular diseases (10). In fact, different from this study, patients enrolled in MESA did not have a history of cardiovascular diseases. In this study, patients with both DM and coronary calcification were enrolled explicitly based on two facts. First, FGF-21 played an essential role in glucose metabolism. For example, FGF-21 could regulate insulin sensitivity. Second, FGF-21 was critical in vessel calcification. Also, in MESA, the FGF-21 level was not related to the incidence of atrial fibrillation during follow- 
Table 1 Baseline characteristics in general and subgroups

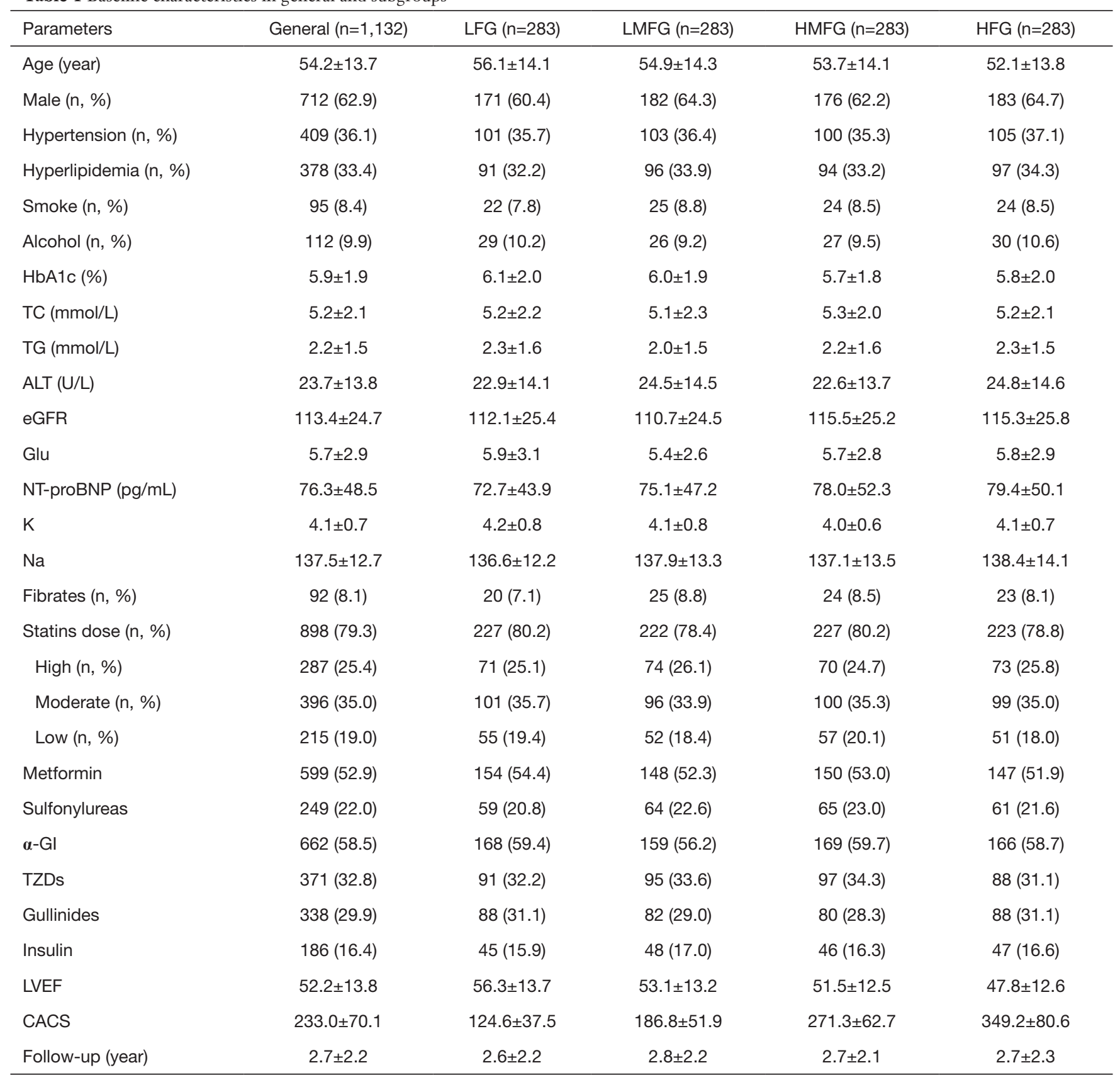

LFG low-FGF-21 group; LMFG, lower-medium-FGF-21 group; HMFG, higher-medium-FGF-21 group; HFG, high-FGF-21 group; TC, total cholesterol; TG, triglyceride; ALT, alanine aminotransferase; $\alpha$-GI, $\alpha$-Glycosidase inhibitors; CACS, coronary artery calcification score; LVEF, left ventricular ejection fraction; TC, total cholesterol; TG, triglyceride; TZDs, thiazolidinediones.

up (11). With these two findings in MESA, FGF-21 indeed should not be used as a predictor of disease or prognosis in a general population. Otherwise, in patients with HF, whether systolic or diastolic HF, studies have found that a higher plasma level of FGF-21 predicted worse heart function
$(12,13)$. In another study aimed at patients with DM, the serum level of FGF-21 was a predictor of CHD (14). However, in patients with end-stage renal disease, circulating FGF-21 was related to mortality, but not suitable to predict a cardiovascular event. However, this 
Table 2 Results at 1-year follow-up

\begin{tabular}{|c|c|c|c|c|c|}
\hline Parameters & General $(n=1,132)$ & LFG $(n=283)$ & LMFG $(n=283)$ & HMFG $(n=283)$ & HFG $(n=283)$ \\
\hline ALT & $24.1 \pm 14.2$ & $23.2 \pm 13.8$ & $21.6 \pm 14.7$ & $24.7 \pm 13.9$ & $22.5 \pm 14.3$ \\
\hline eGFR & $95.7 \pm 21.9$ & $109.2 \pm 24.7$ & $99.4 \pm 23.1$ & $91.3 \pm 23.5$ & $82.7 \pm 21.6$ \\
\hline Glu & $5.7 \pm 2.9$ & $5.9 \pm 3.1$ & $5.4 \pm 2.6$ & $5.7 \pm 2.8$ & $5.8 \pm 2.9$ \\
\hline $\mathrm{TG}$ & $2.2 \pm 1.5$ & $2.3 \pm 1.6$ & $2.0 \pm 1.5$ & $2.2 \pm 1.6$ & $2.3 \pm 1.5$ \\
\hline $\mathrm{K}$ & $4.2 \pm 0.8$ & $4.3 \pm 0.8$ & $4.1 \pm 0.9$ & $4.2 \pm 0.7$ & $4.2 \pm 0.8$ \\
\hline $\mathrm{Na}$ & $136.8 \pm 11.9$ & $138.2 \pm 12.6$ & $137.5 \pm 12.8$ & $135.2 \pm 13.1$ & $136.3 \pm 11.2$ \\
\hline $\mathrm{HbA1c}$ & $5.9 \pm 1.9$ & $6.1 \pm 2.0$ & $6.0 \pm 1.9$ & $5.7 \pm 1.8$ & $5.8 \pm 2.0$ \\
\hline
\end{tabular}

LFG low-FGF-21 group; LMFG, lower-medium-FGF-21 group; HMFG, higher-medium-FGF-21 group; HFG, high-FGF-21 group; ALT, alanine aminotransferase; TC, total cholesterol; TG, triglyceride; LVEF, left ventricular ejection fraction.

Table 3 MACEs in follow-up

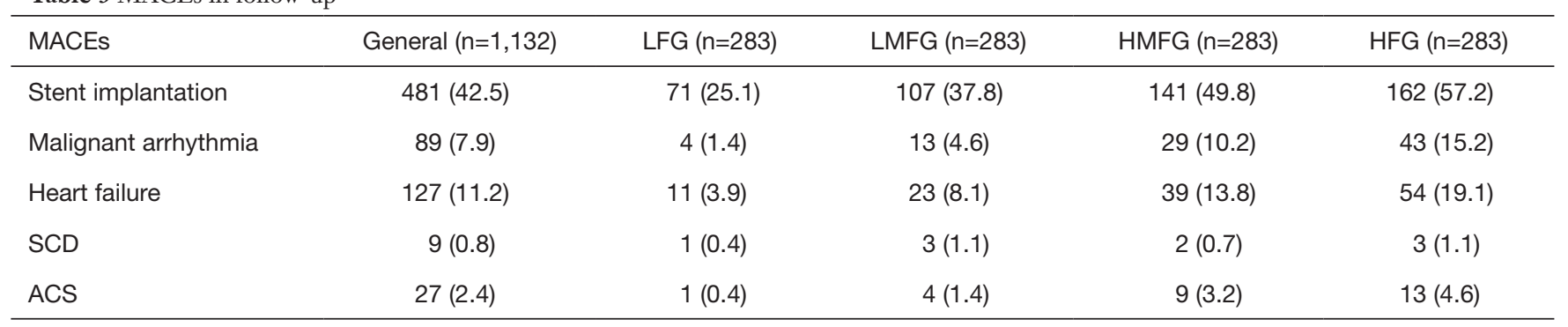

MACEs, major adverse cardiovascular events; LFG low-FGF-21 group; LMFG, lower-medium-FGF-21 group; HMFG, higher-medium-

FGF-21 group; HFG, high-FGF-21 group; SCD, sudden cardiac death; ACS, acute coronary syndrome.

study suggested that FGF-21 could predict the long-term prognosis in specific patients, such as those with both DM and coronary calcification. In studies with positive results, a higher level of FGF-21 predicted worse prognosis (15-17).

Coronary calcification is a risk factor of poor prognosis, especially in patients with cardiovascular disease and related disease or status such as DM. With the increase in CACS from 0 to 1,000 , the 15 -year general mortality increases from $3 \%$ to $28 \%$ simultaneously $(18,19)$. However, in the real world of PCI, CAC significantly increases the difficulty and expenditure of operation (20,21). Moreover, the long-term history of DM increases the likelihood of calcification of the main coronary artery and the inclination of atherosclerosis in the small coronary artery (22). PCI is more challenging to operate when ischemia occurs in patients with a long-term history of DM, especially those without well-controlled blood sugar (23-25). The diffused coronary lesions lead to ventricular remodeling and refractory HF $(26,27)$. These patients are also at risk of suffering from malignant arrhythmia, renal dysfunction, and even SCD $(28,29)$. Therefore, patients at an elevated risk should be recognized at the earliest. In this study, the lower baseline serum level of FGF-21 correlated with lower CACS in patients with DM and CAC. However, earlier studies showed that FGF-21 alleviated vessel calcification through multiple mechanisms (6-9). It is reasonable to assume that a higher level of FGF-21 predicts lighter vessel calcification. This study presented results contradictory to this hypothesis. The reason for this contradiction was related to several possibilities; for example, the possibility that some negative feedback existed between FGF-21 expression and coronary calcification. In other words, coronary calcification could induce the expression of FGF21. Further studies should investigate this possibility in vivo 
and in vitro.

After enrollment, 481 patients underwent percutaneous coronary stent implantation due to cardiac ischemia. Subgroup analysis revealed that the group with a higher level of FGF-21 had a higher incidence of stent implantation and rotational atherectomy. This result was consistent with the study by Lee et al. (14). ACS was the dominant reason (389/481, 80.9\%) for patients undergoing PCI in this study. Moreover, the higher the level of FGF-21, the sooner the ACS occurred. These findings suggested that patients with both $\mathrm{DM}$ and CAC were at higher risk of ACS when a higher level of FGF-21 was confirmed. These patients should receive intensive medication therapy and follow-up monitoring. At the same time, a higher level of FGF-21 was related to malignant arrhythmia, HF, and SCD. The main reason involved in this phenomenon should be myocardial ischemia and diabetic automatic nerve disease. Further studies are needed to confirm this hypothesis.

The relationship between glucose-lower therapy or lipidlower therapy and serum level of FGF-21 is still unclear. In the present study, the medication in four subgroups was compared, and no significant difference was found. Earlier findings were also controversial. In some studies, metformin could decrease the expression level of FGF-21, while in other, studies the results were vice versa (30-32). In a word, several problems related to FGF-21 still need to be solved.

\section{Acknowledgments}

Funding: None.

\section{Footnote}

Conflicts of Interest: The authors have no conflicts of interest to declare.

Ethical Statement: The authors are accountable for all aspects of the work in ensuring that questions related to the accuracy or integrity of any part of the work are appropriately investigated and resolved. This study was approved the Ethics Committee of Beijing Aerospace General Hospital (No. 2003-02).

Open Access Statement: This is an Open Access article distributed in accordance with the Creative Commons Attribution-NonCommercial-NoDerivs 4.0 International License (CC BY-NC-ND 4.0), which permits the non- commercial replication and distribution of the article with the strict proviso that no changes or edits are made and the original work is properly cited (including links to both the formal publication through the relevant DOI and the license). See: https://creativecommons.org/licenses/by-nc-nd/4.0/.

\section{References}

1. Singhal G, Fisher FM, Chee MJ, et al. Fibroblastgrowth factor 21 (FGF21) protects against high fat diet induced inflammation andislet hyperplasia in pancreas. PLoS One 2016;11:e0148252.

2. Shan Z, Alvarez-Sola G, Uriarte I, Arechederra M, et al. Fibroblast growth factors 19 and 21 in acute liver damage. Ann Transl Med 2018;6:257.

3. Kokkinos J, Tang S, Rye KA, et al. The role of fibroblast growth factor 21 in atherosclerosis. Atherosclerosis 2017;257:259-65.

4. Zhang W, Chu S, Ding W, et al. Serum Level of Fibroblast Growth Factor 21 Is Independently Associated with Acute Myocardial Infarction. PLoS One 2015;10:e0129791.

5. Kiramijyan S, Ahmadi N, Isma'eel H, et al. Impact of coronary artery calcium progression and statin therapy on clinical outcome in subjects with and without diabetes mellitus. Am J Cardiol 2013;111:356-61.

6. Shi Y, Lu W, Hou Y, et al. Fibroblast growth factor 21 ameliorates vascular calcification by inhibiting osteogenic transition in vitamin D3 plus nicotine-treated rats. Biochem Biophys Res Commun 2018;495:2448-55.

7. Shi Y, Wang S, Peng H, et al. Fibroblast Growth Factor 21 Attenuates Vascular Calcification by Alleviating Endoplasmic Reticulum Stress Mediated Apoptosis in Rats. Int J Biol Sci 2019;15:138-47.

8. Cao F, Liu X, Cao X, et al. Fibroblast growth factor 21 plays an inhibitory role in vascular calcification in vitro through OPG/RANKL system. Biochem Biophys Res Commun 2017;491:578-86.

9. Liu X, Cao F, Liu S, et al. BMP2/Smad signaling pathway is involved in the inhibition function of fibroblast growth factor 21 on vascular calcification. Biochem Biophys Res Commun 2018;503:930-7.

10. Ong KL, Campbell S, Wu BJ, et al. Relationship of fibroblast growth factor 21 with subclinical atherosclerosis and cardiovascular events: Multi-Ethnic Study of Atherosclerosis. Atherosclerosis 2019;287:46-53.

11. Hui TH, McClelland RL, Allison MA, et al. The relationship of circulating fibroblast growth factor 21 levels with incident atrial fibrillation: The Multi-Ethnic 
Study of Atherosclerosis. Atherosclerosis 2018;269:86-91.

12. RH Chou, PH Huang, CY Hsu, et al. Circulating fibroblast growth factor 21 is associated with diastolic dysfunction in heart failure patients with preserved ejection fraction. Sci Rep 2016;6:33953.

13. Wu CH, Chou RH, Kuo CS, et al. Circulating Fibroblast Growth Factor 21 is Associated with Subsequent Renal Injury Events in Patients Undergoing Coronary Angiography. Sci Rep 2018;8:12425.

14. Lee CH, Woo YC, Chow WS, et al. Role of Circulating Fibroblast Growth Factor 21 Measurement in Primary Prevention of Coronary Heart Disease Among Chinese Patients With Type 2 Diabetes Mellitus. J Am Heart Assoc 2017. doi: 10.1161/JAHA.116.005344.

15. Kohara M, Masuda T, Shiizaki K, et al. Association between circulating fibroblast growth factor 21 and mortality in end-stage renal disease.PLoS One 2017;12:e0178971.

16. Knott ME, Minatta JN, Roulet L, et al. Circulating Fibroblast Growth Factor 21 (Fgf21) as Diagnostic and Prognostic Biomarker in Renal Cancer. J Mol Biomark Diagn 2016. pii: 015.

17. Lee CH, Hui EY, Woo YC, et al. Circulating fibroblast growth factor 21 levels predict progressive kidney disease in subjects with type 2 diabetes and normoalbuminuria. J Clin Endocrinol Metab 2015;100:1368-75.

18. Strauss HW, Nakahara T, Narula N, et al. Vascular Calcification: The Evolving Relationship of Vascular Calcification to Major Acute Coronary Events. J Nucl Med 2019;60:1207-12.

19. Shaw LJ, Giambrone AE, Blaha MJ, et al. Long-Term Prognosis After Coronary Artery Calcification Testing in Asymptomatic Patients: A Cohort Study. Ann Intern Med 2015;163:14-21.

20. Kobayashi Y, Okura H, Kume T, et al. Impact of target lesion coronary calcification on stent expansion. Circ J 2014;78:2209-14.

21. Bourantas CV, Zhang YJ, Garg S, et al. Prognostic implications of coronary calcification in patients with obstructive coronary artery disease treated by percutaneous coronary intervention: a patient-level pooled analysis of 7 contemporary stent trials. Heart 2014;100:1158-64.

22. Rodríguez-Puyol D. Cano-Megías M, Bouarich H, et al. Coronary artery calcification in patients with diabetes mellitus and advanced chronic kidney disease. Endocrinol
Diabetes Nutr 2019;66:297-304.

23. Godoy LC, Rao V, Farkouh ME. Coronary

Revascularization of Patients With Diabetes Mellitus in the Setting of Acute Coronary Syndromes. Circulation 2019;140:1233-5.

24. Brush JE Jr, Siraj ES, Kemp CD, et al. Effect of Diabetes Mellitus on Complication Rates of Coronary Artery Bypass Grafting. Am J Cardiol 2019;124:1389-96.

25. Bates ER. Antiplatelet Therapy in Patients with Coronary Disease and Type 2 Diabetes. N Engl J Med 2019;381:1373-5.

26. Patsouras A, Farmaki P, Garmpi A, et al. Screening and Risk Assessment of Coronary Artery Disease in Patients With Type 2 Diabetes: An Updated Review. In Vivo 2019;33:1039-49.

27. Chen HF, Ho CA, Li CY. Risk of heart failure in a population with type 2 diabetes versus a population without diabetes with and without coronary heart disease. Diabetes Obes Metab 2019;21:112-9.

28. Jiménez-Navarro MF, López-Jiménez F, Barsness G, et al. Long-term prognosis of complete percutaneous coronary revascularisation in patients with diabetes with multivessel disease. Heart 2015;101:1233-9.

29. Nasri H, Rafieian-Kopaei M. Diabetes mellitus and renal failure: Prevention and management. J Res Med Sci 2015;20:1112-20.

30. Kim KH, Jeong YT, Kim SH, et al. Metformin-induced inhibition of the mitochondrial respiratory chain increases FGF2 1 expression via ATF4 activation. Biochem Biophys Res Commun 2013;440:76-81.

31. Zhang M, Liu Y, Xiong ZY, et al. Changes of plasma fibroblast growth factor-21 (FGF-21) in oral glucose tolerance test and effects of metformin on FGF-21 levels in type 2 diabetes mellitus. Endokrynol Pol 2013;64:220-4.

32. Yang M, Dong J, Liu H, et al. Effects of short-term continuous subcutaneous insulin infusion on fasting plasma fibroblast growth factor-21 levels in patients with newly diagnosed type 2 diabetes mellitus. PLoS One 2011;6:e26359.

Cite this article as: Gan F, Huang J, Dai T, Li M, Liu J. Serum level of fibroblast growth factor 21 predicts long-term prognosis in patients with both diabetes mellitus and coronary artery calcification. Ann Palliat Med 2020;9(2):368-374. doi: 10.21037/apm.2020.03.28 